\title{
Biliopancreatic Diversion Versus Gastric Bypass in the Surgical Treatment of Type 2 Diabetes Mellitus in Patients with Body Mass Index Below 35
}

\author{
MOHAMED SHETIWY, M.D.; NASHAT ABD EL-RAZEK, M.D.; HOSAM EL-GHADBAN, M.D., M.R.Cs.; \\ ASHRAF ABBAS, M.D.; AHMED NEGM, M.D.; MOHAMED SAMIR, M.D., M.R.Cs.; \\ MAGDY BASHEER, M.D.; ABD EL-RAHMAN EL-BAHY, M.D. and ASHRAF SHOMA, M.D., F.R.Cs.
}

The Department of General Surgery, Faculty of Medicine, Mansoura University, Mansoura, Egypt

\begin{abstract}
Background: Type 2 Diabetes Mellitus (T2DM) is an important health problem worldwide with about $23 \%$ of patients with morbid obesity have type $2 \mathrm{DM}$. Bariatric surgery originally developed solely as a weight-reduction therapy, but has been found to improve T2DM and to reduce rates of chronic vascular disease and death. The International Diabetes Federation have recommended consideration of bariatric surgery for control of T2DM.
\end{abstract}

Methods: A total number of 45 patients were randomized into two groups, the first group with 26 patients $(n=26)$ were submitted for Biliopancreatic Diversion (BPD) and the second group with 19 patients $(n=19)$ were planned for Roux-en-Y Gastric Bypass (RYGBP). The main objective was to compare the efficacy of bariatric surgery in inducing clinical remission of T2DM mellitus in patients with body mass index less than 35 in both groups.

Results: The operations were performed laparoscopically in all patients. There were no conversions, operative complications or mortality. Data were collected during a two-year follow-up period and were analyzed at the following periods; pre-operatively and post-operatively at 1 months, 4 months, at 8 months, at 1 year and 2 years. The analyzed parameters included the levels of BMI, FBG, HbA1c, Triglycerides and cholesterol. There were no statistical significant difference between the two groups regarding the BMI, mean glucose levels, and $\mathrm{HbA} 1 \mathrm{c}$ levels. However the levels of triglycerides were higher in the BPD group, while the levels of cholesterol were higher in the RYGBP group during most of the followup periods.

Regarding the diabetes outcome, there was significant reduction of patients who showed diabetes remission at one year and those who remains remittent at 2 year, while the number of patients who showed diabetes failure tripled at 2 years follow-up.

Conclusions: Although, BPD is known to have a better effect on type $2 \mathrm{DM}$ in the higher BMI group. There was no difference regarding glycemic control between BPD and RYGBP. And both operations failed to maintain glycemic control at long term follow-up periods.

Correspondence to: Dr. Mohamed Shetiwy, E-Mail:m.shetiwy@yahoo.com
Key Words: $B P D-R Y G B P-$ Metabolic surgery - Surgical treatment of T2DM - Low BMI.

\section{Introduction}

TYPE 2 Diabetes Mellitus (T2DM) is an important health problem that has increased exponentially over the world as a result of increasing the prevalence of obesity. The prevalence of type 2 diabetes mellitus is rapidly increasing worldwide, in parallel with the current obesity epidemic. In 2015, the world wide prevalence of T2DM was estimated at $8.3 \%$ of the adult population, and is expected to rise to $9.9 \%$ by the year 2030 . Morever about $23 \%$ of patients with morbid obesity have T2DM. The worldwide diabetes cost was estimated to be at least $\$ 376$ billion in 2010 and is expected to rise to $\$ 490$ billion by 2030 [1]

Although, bariatric surgery originally developed solely as a weight-reduction therapy, it has been proven that it improves T2DM and to reduces the rates of CVD and death. Furthermore, experimental studies in animals and humans suggest that certain bariatric procedures may improve diabetes control through mechanisms other than weight loss. The International Diabetes Federation have recommended considering bariatric surgery for control of T2DM [2]

\section{Patients and Methods}

Data of 45 patients were collected after randomisation and those patients were operated between July 2012 - June 2014 in San Martino Hospital in Genoa University, Italy and followed-up for two years during a two year joint supervision mission.

A total number of 45 patients were randomized into two groups, the first group with 26 patients 
$(\mathrm{n}=26)$ were submitted for Biliopancreatic Diversion (BPD) and the second group with 19 patients $(\mathrm{n}=19)$ were planned for Roux-en-Y Gastric Bypass (RYGBP).

\section{Inclusion criteria:}

Patients with BMI less than 35 and a duration of diabetes $\geq 3$ years with poor glycemic control (i.e., $\mathrm{HbA} 1 \mathrm{c} \geq 7.5 \%$ ) in spite of hypoglycemic therapy in accordance with Good Clinical Practice (GCP) were invited to participate in this study.

Medical history carefully obtained from each patient and through physical examination was done, and fully eligible patients were asked to provide a written informed consent.

Twenty six patients were planned for Biliopancreatic Diversion (BPD). A distal two-third gastrectomy was carried out, aiming at leaving about $400 \mathrm{ml}$ of gastric remnant. The gastrointestinal continuity was re-established by dividing the small bowel $300 \mathrm{~cm}$ proximal to the ileocecal valve, closure of the intestinal stumps, and connecting the proximal one end-to-side to the distal ileum at $50 \mathrm{~cm}$ from the ileocecal valve. The distal stump of the transected bowel was anastomosed to the left corner of the gastric stump, preferably in a totally hand-sewn fashion. However, any gastroenterostomy technique was allowed, according to the surgeon's preference. Cholecystectomy was considered a part of this operation.

Nineteen patients were planned for Roux-enY Gastric Bypass (RYGBP): A gastric pouch with a $30 \pm 10 \mathrm{ml}$ capacity was created on a nasogastric calibrating tube $(36 \mathrm{~F})$ by sectioning the stomach with a linear stapler $(4 \mathrm{~cm}$ on the lesser curve horizontally, $4 \mathrm{~cm}$ distal to the esophagogastric junction, and then vertically until reaching of the angle of Hiss). Treitz ligament was identified, then the jejunum was transected (at $100 \mathrm{~cm}$ from the ligament of Treitz) and the two stumps were closed. The distal stump was anastomosed to the distal end of the gastric pouch. The preferred gastrojejunal anastomosis was totally hand-sewn. Finally, the proximal stump of the transected bowel was joined end-to-side to the jejunum $(150 \mathrm{~cm}$ distal to the gastroenterostomy).

\section{Statistical analysis:}

Analysis of data was performed using Statistical Package for Scientific Studies (SPSS) V.22 for Windows 7. Numerical data was expressed as means \pm Standard Deviation (SD) and comparisons between groups were done. The significance of the test results was assessed in the form of $p$-value that was considered significant when $p$-value was $\leq 0.05$.

For comparing between the two groups an independent samples $t$-test was used while paired $t$ test was used to test the presence of significant effect of surgery on different variables measured in the study. For measuring and comparing the diabetic outcome in both groups a Chi square analysis was carried out to determine significant effect.

\section{Results}

The patients recruited were 30 males $(66.6 \%)$ and 15 females $(33.3 \%)$. The BPD group (group 1 ) included 26 patients $(n=26)$ and the RYGBP group (group 2) included 19 patients $(n=19)$. The two groups were matched for age, sex, pre-operative $\mathrm{BMI}$, duration of diabetes, pre-operative glucose levels and $\mathrm{HbA} 1 \mathrm{c}$ levels showing no statistical significance (Table 1).

Table (1): Comparisons between patient base line characteristics.

\begin{tabular}{lllll}
\hline & $\begin{array}{c}\text { Type of } \\
\text { operation }\end{array}$ & No & $\begin{array}{c}\text { Mean } \\
\pm S D\end{array}$ & $\begin{array}{c}p- \\
\text { value }\end{array}$ \\
\hline • Age (years). & BPD & 26 & $53.6 \pm 11$ & 0.363 \\
& RYGBP & 19 & $56.5 \pm 9.5$ & \\
• Pre-operative BMI & BPD & 26 & $31.2 \pm 2.9$ & 0.057 \\
$($ Kg/m). & RYGBP & 19 & $32.9 \pm 1.4$ & \\
• Pre-operative Glucose & BPD & 26 & $217 \pm 62$ & 0.501 \\
$($ Mg/dl). & RYGBP & 19 & $204 \pm 59$ & \\
- Pre-operative HbA1c & BPD & 26 & $9.3 \pm 1.4$ & 0.894 \\
$(\%)$. & RYGBP & 18 & $9.3 \pm 1.9$ & \\
- Duration of diabetes & BPD & 26 & $12 \pm 6$ & 0.774 \\
(years). & RYGBP & 19 & $12 \pm 5.6$ & \\
\hline
\end{tabular}

Early post-operative complications:

The operations were performed laparoscopically in all patients. There were no conversions or operative complications, nor mortality. Early postoperative complications were one case $(2.2 \%)$ from the BPD group who developed bleeding and was explored in the first post-operative day and another case $(2.2 \%)$ in the RYGBP group with cirrhotic liver who developed ascites two months after the operation. Two cases $(4.4 \%)$ one from each group developed gastroplegia that resolved within 2 weeks.

\section{Late post-operative complications:}

Late complications in the BPD group included; three cases $(6.7 \%)$ developed severe diarrhea and perianal excoriation. One of them asked for revision and was revised in the first follow-up year and 
therefore was excluded from the analysis and another one who was revised in the second year and his data was included in the analysis. The third patient responded well to medical treatment and was not revised. Additionally, in the BPD group, one patient developed proteinuria in the 2 nd postoperative year, while another one had port-site incisional hernia and had hernia repair at another hospital without complications and a third had acute leukemia with pulmonary infiltrates and died after 2 years.

In the RYGBP group three patients suffered from anemia and severe upper abdominal pain. Upper GIT endoscopy showed post-anastomotic ulcer in two patients.

\section{Follow-up evaluations:}

Data were collected and were analyzed at the following periods; pre-operatively, at 1 months, at 4 months, at 8 months, at 1 year and 2 years postoperative. The analyzed parameters included the levels of BMI, FBG, $\mathrm{HbA} 1 \mathrm{c}$, triglycerides and cholesterol.

Significant reduction of preoperative BMI (mean=31.9 \pm 2.5 ) occurred after 4 months, continued throughout the first year (mean=25.2 \pm 2.3 ) and remain stable at (mean $=25.8 \pm 2.6)$ till the end of the second year Fig. (1). Regarding BMI reduction, there was no statistical significant difference was observed between BPD and RYGBP groups except in the second year in favor of RYGBP.

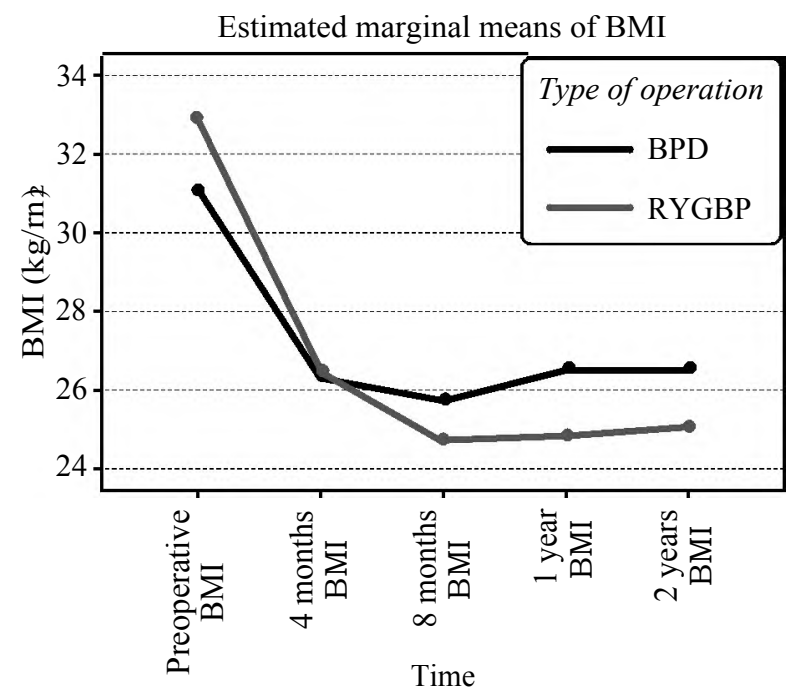

Fig. (1): BMI changes in both groups during the follow-up period.

Glucose levels were significantly reduced between the pre-operative fasting glucose level (mean $=211.8 \pm 60.8 \mathrm{mg} / \mathrm{dl}$ ) than for the 1 year level $(m e a n=150.8 \pm 55.7 \mathrm{mg} / \mathrm{dl})(p<0.001)$, and for the
2 year level (mean=148.9 \pm 54.1$)(p<0.001)$ Fig. (2). Regarding comparisons between mean fasting blood glucose in two groups, the analysis showed that fasting blood glucose levels were not significantly different between the two groups.

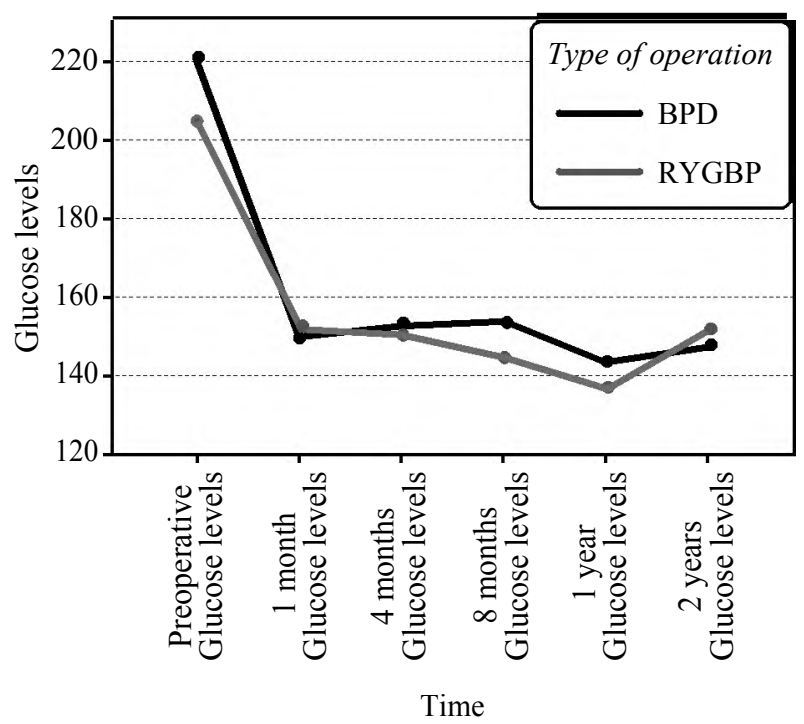

Fig. (2): Changes in glucose levels in both groups during the follow-up period.

Regarding the changes in $\mathrm{HbA1c}$ in both groups, data showed that its levels were significantly reduced from a pre-operative $\mathrm{HbA1c}($ mean $=9.3 \pm 1.6)$ to a 1 year $\mathrm{HbA} 1 \mathrm{c}($ mean $=6.7 \pm 1.4)(p<0.0001)$, and a 2 year $\mathrm{HbA} 1 \mathrm{c}($ mean $=7 \pm 1.4)(p<0.0001)$ Fig. (3). Regarding comparisons between $\mathrm{HbAlc}$ in two groups' analysis indicated that $\mathrm{HbA} 1 \mathrm{c}$ levels were significantly lower for the BPD group only in the second year follow-up than for RYGBP group. No significant differences were seen between the two groups by the end of the fifth year where the levels of HbA1c in the BPD group rose approaching that of the RYGBP group.

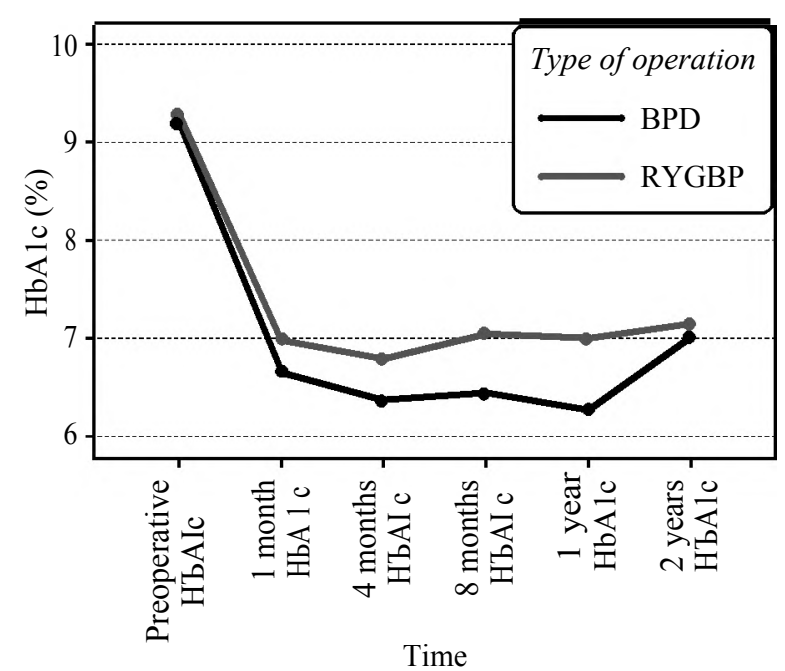

Fig. (3): Levels of HbA1c in both groups during the followup period. 
Regarding the changes in triglycerides in both groups, analysis showed that triglycerides levels were significantly higher for the pre-operative triglycerides $($ mean $=205 \pm 129)$ than for the 1 year triglycerides $($ mean $=158 \pm 94)(p=0.019)$, and for the 2 year triglycerides (mean $=155 \pm 89)(p=0.028)$ Fig. (4). Comparing the triglycerides levels in both groups indicated that triglycerides levels were significantly higher for the BPD group in the $8^{\text {th }}$ month, in the first year follow-up and in the second year follow-up than for RYGBP group.

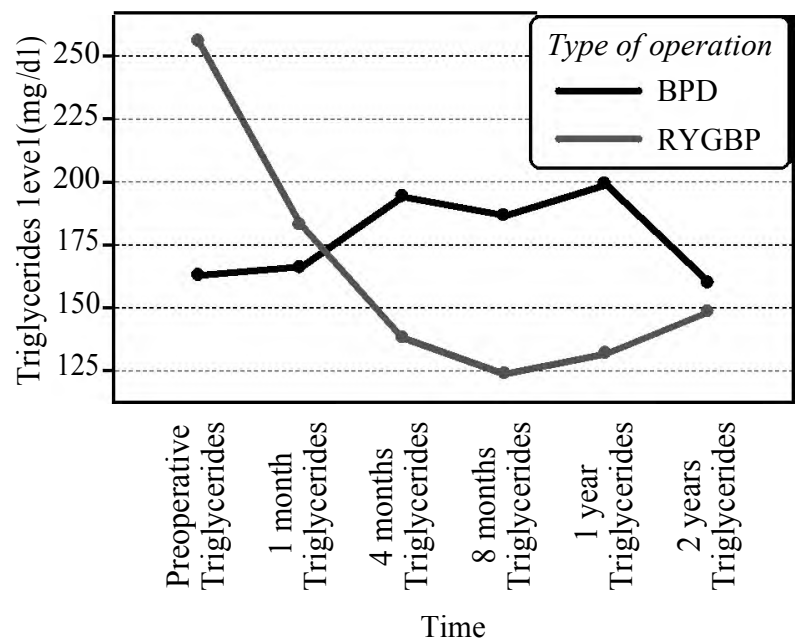

Fig. (4): Effect on triglycerides in both groups in the followup period

Total cholesterol levels indicated that scores were significantly higher for the preoperative total cholesterol $($ mean $=205 \pm 129)$ than for the 1 year total cholesterol (mean $=158 \pm 94),(p=0.019)$, and for the 2 year total cholesterol (mean $=155 \pm 89)(p=$ 0.028 ) Fig. (5). Comparing total cholesterol in both groups, results indicated that total cholesterol levels were significantly lower for the BPD group than for RYGBP group in all follow-ups.

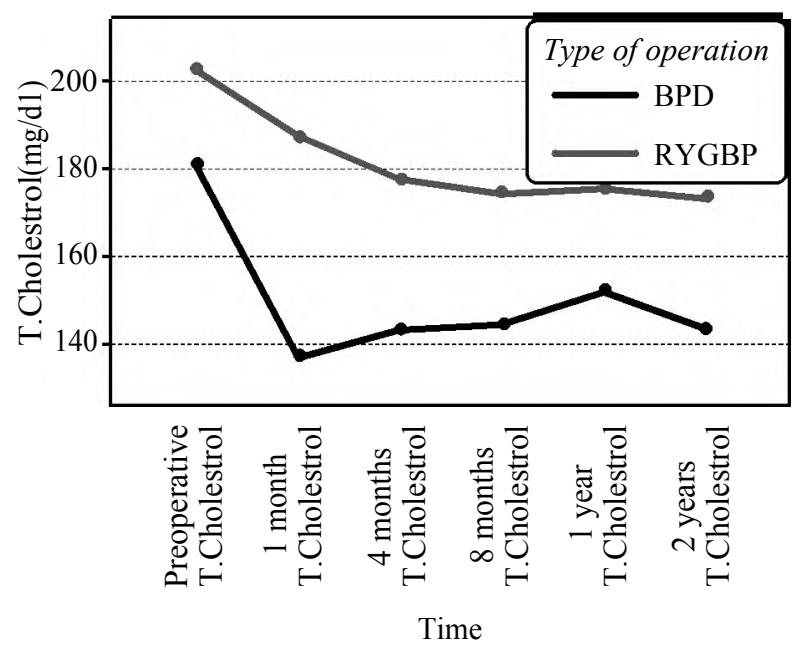

Fig. (5): Effect on cholesterol levels.

\section{Diabetes outcome:}

Patients' responses were measured at 1 and 2 years and then classified depending on their antidiabetic treatment and $\mathrm{HbA} 1 \mathrm{c}$ levels into the following 4 groups:

- Diabetes full remission (HbA1c 6\% or below, on free diet and with no anti-diabetic medical therapy).

- Diabetes control (HbA1c between 7\% and 6.1\%, under the same conditions).

- Diabetes improvement (stable reduction of preoperative $\mathrm{HbA} 1 \mathrm{c}$ by at least $1 \%$, with less antidiabetic therapy).

- Failure (none of the above).

Analysis of the data at 1 and 2 years followup showed that patients who were classified as diabetes full remission in the first year were 34 patients $(75.5 \%), 21(46.6 \%)$ in the BPD group and 13 patients $(28.8 \%)$ in the RYGBP group. After two years, this number decreased to 11 patients (24.4\%), $6(13.3 \%)$ in the BPD group and $5(11.1 \%)$ patients in the RYGBP group.

While only 3 patients $(6.6 \%)$ achieved diabetes control at one year, one patient in the BPD group $(2.2 \%)$ and 2 in the RYGBP $(4.4 \%)$, this number increases to 7 patients (15.5\%), 4 in the BPD group $(8.8 \%)$ and 3 in the RYGBP $(6.6 \%)$ in the second year follow-up.

Only 4 patients $(8.8 \%)$ showed diabetes improvement at one year 1 in the BPD group $(2.2 \%)$ and 3 in RYGBP group (6.6\%), and also rises to 16 patients $(35.5 \%)$ showing diabetes improvement 9 in the BPD group (20\%) and 7 in RYGBP group $(15.5 \%)$.

While only 3 patients $(6.6 \%)$ showed failure at 1 year, 2 in the BPD group (4.4\%) and 1 in the RYGBP group $(2.2 \%)$, they became 10 patients $(22.2 \%)$ showing failure at 2 years, 6 in the BPD group (13.3\%) and 4 in the RYGBP group (8.8\%). Comparing the two groups on diabetes outcome showed no statistical significance between both groups Figs. $(6,7)$.

\section{Predictors of outcome:}

In order to predict the patients who will become remitters we divided the patients according BMI, age, duration of diabetes, pre-operative insulin therapy, and pre-operative levels of HbAlc. None of them were statistically significant but this may be attributed to unequal samples in both groups, and we think it would be useful to report some of these predictors and there effect on diabetes remission. 


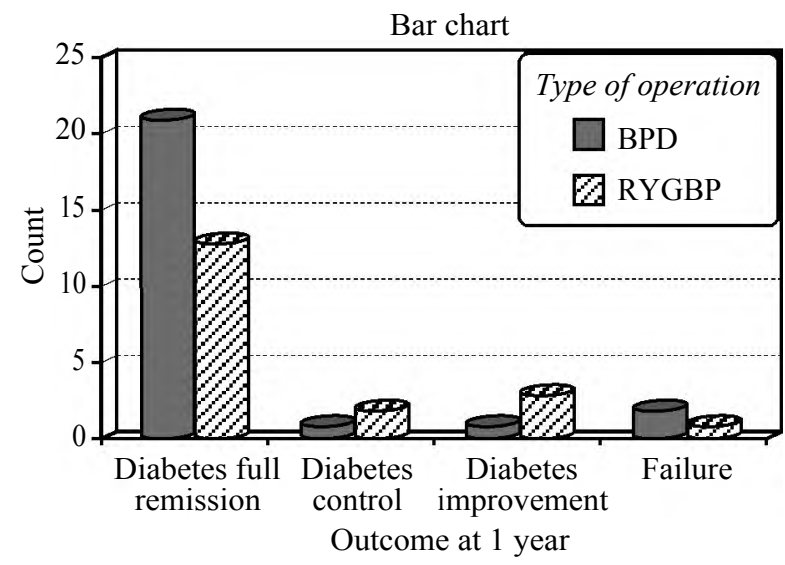

Fig. (6): Diabetes outcome at 1 year.
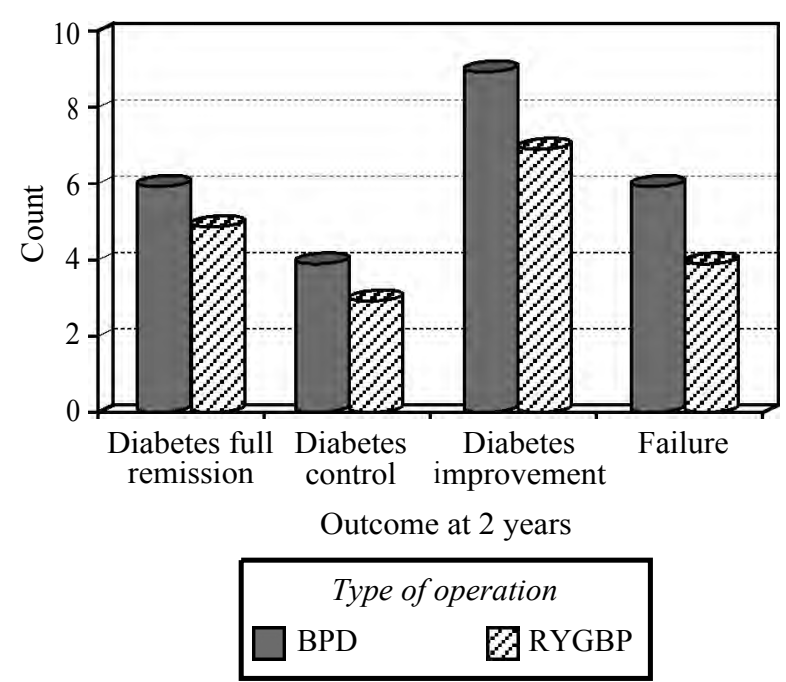

Fig. (7): Diabetes outcome at 2 years.

Regarding the BMI, patients were classified into two groups, 33 patients (75\%) with BMI 3035 , and 11 patients $(25 \%)$ with $\mathrm{BMI}>30$. We found that all the patients with diabetes remission at 2 years, 11 patients $(25 \%)$, were from the group with BMI 30-35, and none of the patients with BMI $<30$ had diabetes full remission at 2 years Fig. (8).

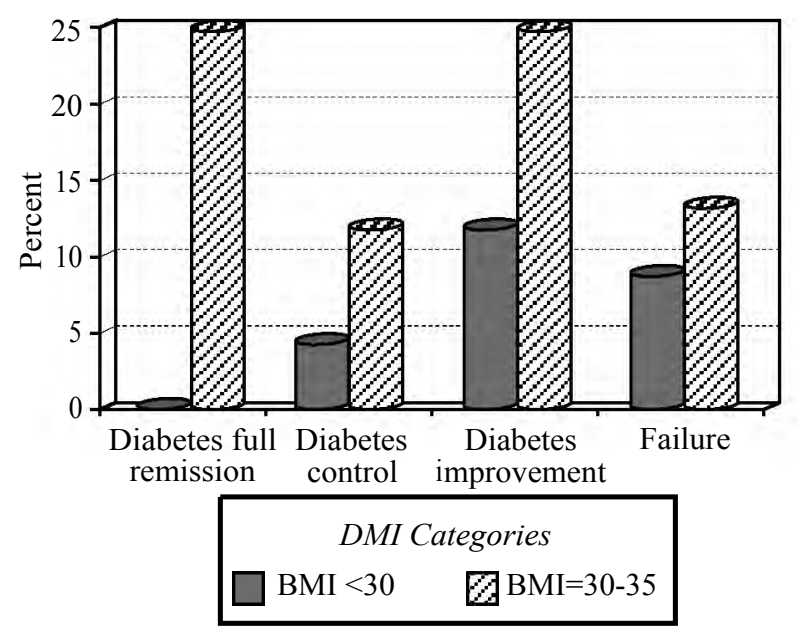

Fig. (8): BMI below or above 30 as a predictor of diabetes full remission at 2 years follow-up.
Regarding the age, patients were divided into two groups the first group had 12 patients $(27.2 \%)$ with age 50 years or less, and the second group of 32 patients $(72.7 \%)$ with age more than 50 years. We found that most patients who fail at 2 years were from the older age group (above 50) Fig. (9).

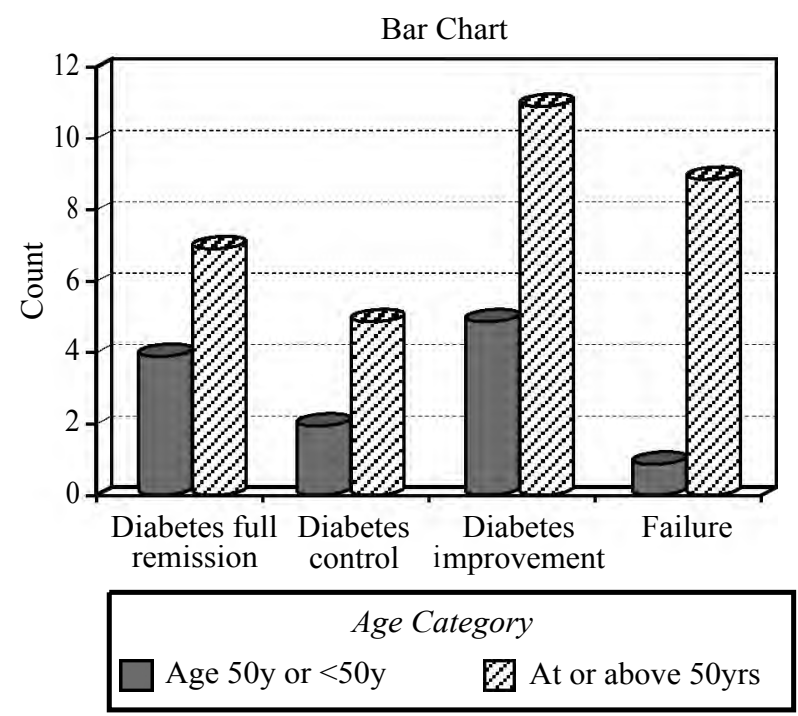

Fig. (9): Age below or above 50 as a predictor of diabetes full remission at 2 years follow-up.

Regarding the preoperative duration of DM. Patients were divided into two groups the first group had 19 patients $(43 \%)$ with 10 years or less duration of diabetes and the second with 25 patients (57\%) with duration more than 10 years. We found that most patients who failed at 2 years were from the group with more than 10 years of duration of DM Fig. (10).
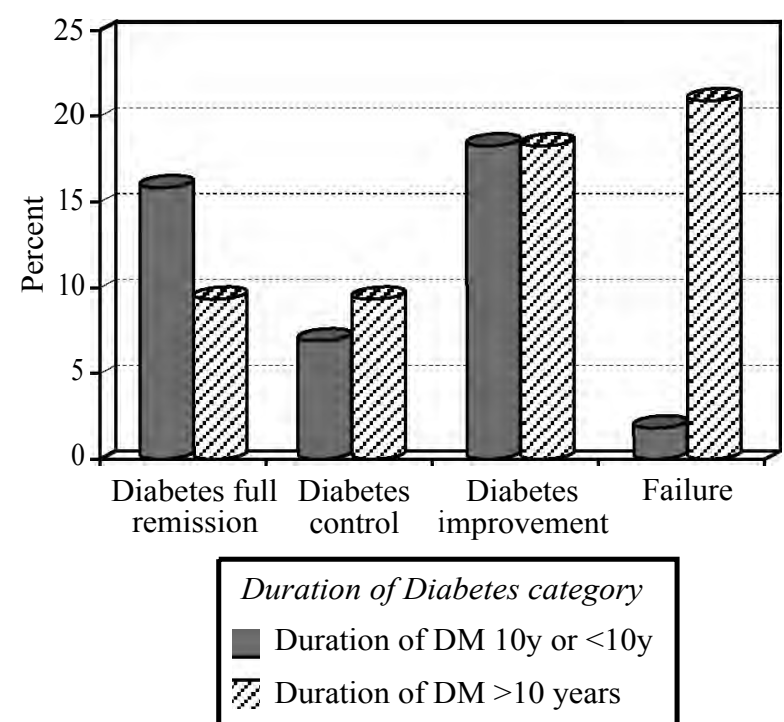

Fig. (10): Duration of diabetes less or more than 10 years as a predictor of Diabetes full remission at 2 years follow-up. 
Patients were divided into two groups, the first with 20 patients $(45 \%)$ who didn't receive insulin, and 24 patients $(55 \%)$ who were given insulin therapy pre-operatively. Patients who take preoperative insulin tend more to fail at 2 years, but with no statistical significance Fig. (11).

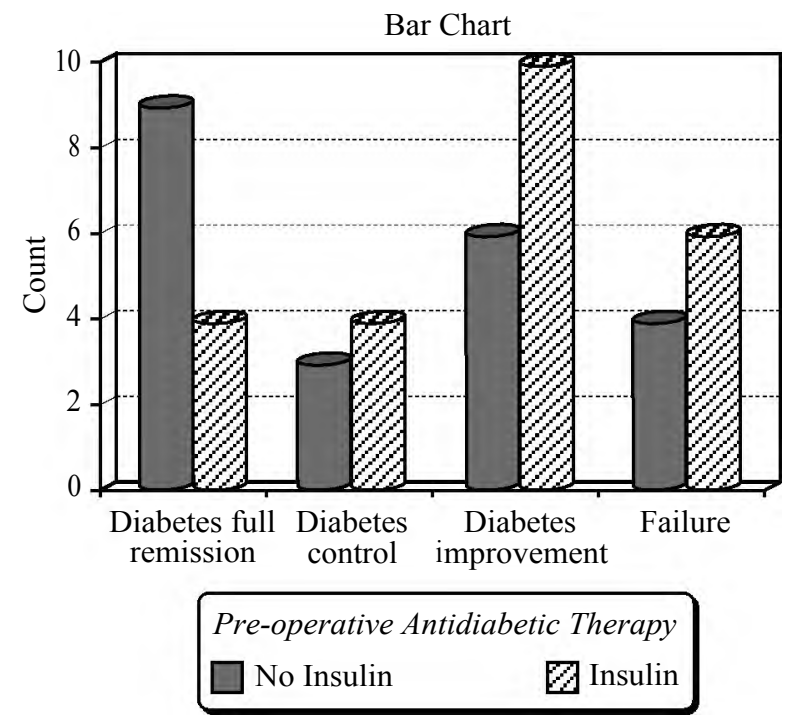

Fig. (11): Pre-operative insulin therapy as a predictor of diabetes full remission at 2 years follow-up.

In order to predict the effect of pre-operative $\mathrm{HbA} 1 \mathrm{c}$ levels, patients were divided into two groups one with 23 patients $(52 \%)$ with $\mathrm{HbA} 1 \mathrm{c}$ level of $9 \%$ or less and the other group with 21 patients $(84 \%)$ with levels more than $9 \%$. Analysis showed that no statistical significance between the two groups, but remission was seen more in patients with levels less than 9\% Fig. (12).

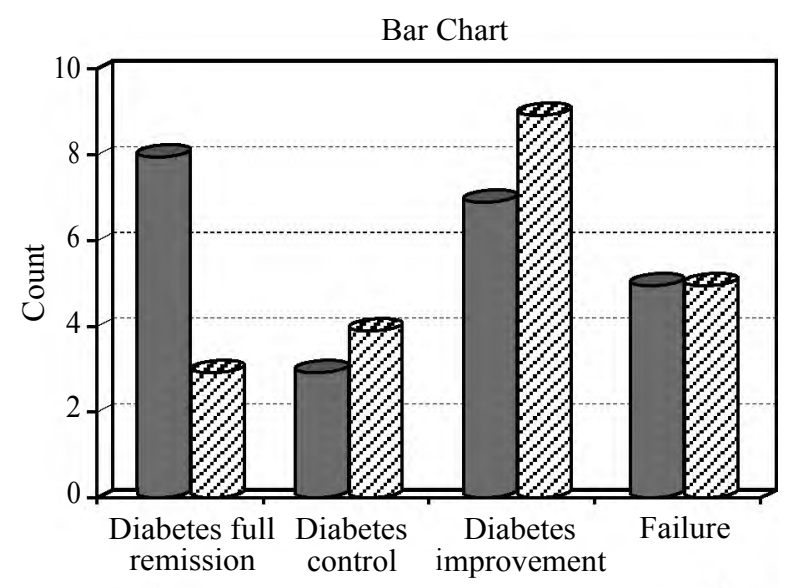

HbAl c pre-operative category

HbAlc 9 or $<9$

Q $\mathrm{HbAlc}>9$

Fig. (12): Pre-operative HbaA1c level below or above 9\% as a predictor of diabetes full remission at 2 years follow-up.

\section{Discussion}

Diabetes type 2 and obesity, sometimes referred to as diabesity, are the 21 th century pandemic. The International Diabetes Federation (IDF) in 2014 has estimated that by the year 2035 approximately 592 million people will be affected by this killer disease all over the world, out of which $90 \%$ will have type 2 diabetes mellitus.

Multiple case-controlled studies demonstrate impressive, sustained improvements in T2DM among patients with severe obesity (BMI > 35 $\mathrm{kg} / \mathrm{m}^{2}$ ) after gastric banding, RYGBP, and BPD. In a meta-analysis involving 136 studies and 22,094 patients, with T2DM resolution defined as persistent normoglycemia without diabetes medications, Buchwald et al., reported an overall $77 \%$ remission of T2DM after bariatric surgery [3]. The remarkable control of diabetes in severely obese patients after bariatric surgery, along with experimental studies showing that gastrointestinal operations can improve diabetes in both obese and nonobese animals, suggests that surgery may be an interesting option for moderately obese or nonobese patients with T2DM [4].

Metabolic surgery to treat T2DM in patients with low BMI show desirable effects as the weight loss was stabilized. In our study, BMI was stabilized in both groups at 1 year, (25.7 for BPD group and 24.7 for RYGBP group) and at 2 years (26.5 for BPD group and 25 for the RYGBP group) with no significant difference between the two groups denoting that these operations in low BMI patients didn't lead to excessive weight loss.

Improvement of diabetic status was significant after metabolic surgery as observed in metanalysis done by Buchwald et al. Discontinuation of antidiabetic medication in $86.8 \%$ and remission in $64.7 \%$ of T2DM patients after metabolic surgery were achieved. It has been described that malabsorptive bariatric procedures have higher diabetes remission rates than restrictive ones [5].

In our study, at diabetes full remission in the first year were 34 patients $(75.5 \%), 21$ from the BPD group (46.6\%) and 13 patients $(28.8 \%)$ from the RYGBP group, and after two years, this number decreases to 11 patients $(24.4 \%), 6$ from the BPD group $(13.3 \%)$ and 5 patients from the RYGBP group (11.1\%).

Diabetes mellitus is a chronic progressive disease, so durability of the treatment option is highly required. Two studies showed durable diabetes remission of T2DM during 5-18 years period after 
mini gastric bypass and BPD $(168,169)$. This durability is tested strongly in our study, and the results were disappointing as the remitters number decreased from 34 patients $(75.5 \%)$ in the first year to $11(24.4 \%)$ patients. While patients showing failure of treatment were only 3 patients $(6.6 \%)$ at one year and the number of failure increases to 10 patients $(22.2 \%)$ after 2 years in both groups, suggesting a very high rate of diabetes relapse over time. And this is different from some studies which focused on patients with mild obesity (BMI 30$35)$, where the results where more encouraging [6]

The mortality rates from bariatric operations (0.28-0.35\%) compare favorably with those of other commonly performed operations, including laparoscopic cholecystectomy, whose mortality in USA ranges between 0.35 and $0.60 \%$ [7]. In our study, no mortality occurred in both groups, except for one case who died after 2 years from acute leukemia, and therefore his data was included in the analysis.

The natural history of type 2 diabetes is also important to consider in determining the timing of intervention. As the diabetes state progresses, there is continued beta-cell deterioration together with a decline in insulin secretion within 6-10 years of T2DM diagnosis [8]. In Our study we included patients with only more than 3 years history of $\mathrm{DM}$ and the mean duration of diabetes in all patients was 12 years.

Interestingly, and differently from what observed as a rule in bariatric surgery series, there was a strong prevalence of men in our study population, which became striking in the lower BMI range. This could be explained by the more frequency of type 2 diabetes in men, even if the difference is only slight.

Results of metabolic surgery in patients with a BMI of $<35$ :

RYGBP was the first operation in morbidly obese patients to be proved effective in inducing T2DM remission. And these results, encouraged some authors to treat T2DM patients with a BMI of $<35$ with RYGB with reported good results in patients with T2DM (181). Lee et al., showed consistently good results in an Asian population after gastric bypass with a significantly successful treatment of T2DM (HbA1c <7\%) in up to $76.5 \%$ of patients 1 year post-operatively (168). Tavares de Sa et al., studied 27 patients with T2DM with a BMI of 30-35 and a mean follow-up of 20 months. They achieved resolution in $48.1 \%$ of patients and diabetes control in $44.5 \%$ patients using definitions similar to the definitions used in our study and $7.4 \%$ only of the group were maintained on medication (182). In an Asian Indian population, Shah et al., reported RYGBP in 15 patients with T2DM and a BMI of 22-35. At 3 months and thereafter, $100 \%$ of patients were euglycemic and no longer required medication [9]

More recently, some Asian groups reported the largest multicenter prospective study to date, which included 200 uncontrolled diabetic patients with BMI $<35 \mathrm{~kg} / \mathrm{m} 2$. Gastric bypass was performed in $172(86 \%)$ patients, whereas $28(14 \%)$ patients had restrictive procedures (sleeve or gastric banding). Diabetes remission was more frequent in the gastric bypass group when compared with the group receiving the restrictive procedures $(79.3$ versus $55.3 \%)$ in patients with early-onset diabetes less than 5 years [10].

In our study regarding the RYGBP group, 19 patients were admitted to surgery and complete remission was seen in 13 patients at 1 year and improvement or control in the other 5 patients and only one patient showed failure to achieve any improvement. This may differ slightly from other studies because we use a more strict definition of DM remission than some other studies and also our patients had a longer duration of diabetes mellitus. And regarding a long term results, it is different from the results mentioned by Cohen in long term follow-up after 6 years, and we think this can be attributed to selection of patients with mild obesity only as we included patients with simple overweight also in the study.

Regarding the biliopancreatic diversion, Chiellini et al., published a report on five patients who underwent BPD and were studied. They concluded that BPD can achieve adequate control of T2DM in patients with a BMI of $<35$. Diabetes remission was seen for one and half year of follow-up after BPD without pharmacological therapy [11]. In our study, the BPD group had 26 patients and at 1 year 21 patients showed complete diabetes remission, but at 2 year follow-up this number decreased to 6 patients. This suggests a high rate of diabetes relapse in patients with low BMI after long term follow-up.

\section{Comparison between RYGBP and BPD:}

The main aim of this study was to compare RYGBP and BPD in the treatment of type 2 diabetes mellitus in low BMI patients. To our knowledge, this is the first study to compare these two operations in this BMI category, but some studies compare between these two operations but in patient 
population with higher BMI. Skroubis et al., compared two groups of patients with BMI 35-50 $\mathrm{kg} / \mathrm{m}^{2}$, one group had RYGBP and the other had a variant of BPD and followed these patients for 8 years. Late results showed that both procedures are safe and effective; BPD seems to prevail in terms of successful weight loss without a significantly higher incidence of metabolic and nonmetabolic complications, but with long-lasting superiority of BPD over RYGBP in the remission of comorbidities [12].

In our study, no statistical significance between the two groups except the effect on lipid profile as BPD leads to a significantly lower cholesterol levels while the levels of triglycerides were significantly higher than that of the RYGBP group.

\section{Conclusion:}

Although, BPD is known to have a better effect on type $2 \mathrm{DM}$ in the higher BMI group. There was no difference regarding glycemic control between BPD and RYGBP. And both operations failed to maintain glycemic control at long term follow-up periods.

\section{References}

1- American Diabetes Association. American Diabetes Association Standards of Medical Care in Diabetes 2017. Diabetes Care, 40 (1): 78-80, 2017.

2- RUBINO F., SHUKLA A., POMP A. and MOREIRA M.: Bariatric, metabolic, and diabetes surgery: What's in a name? Ann. Surg. 2014; 259 (1): 117-122.

3- BUCHWALD H., AVIDOR Y., BRAUNWALD E., DJENSEN M., PORIES W., FAHRBACH K., et al.: Bariatric surgery A Systematic Review and Meta-analysis. J. Am. Med. Assoc., 151 (14): 243-53, 2004.
4- PACHECO D., De LUIS D.A., ROMERO A., GONZÁLEZ SAGRADO M., CONDE R., IZAOLA O., et al.: The effects of duodenal-jejunal exclusion on hormonal regulation of glucose metabolism in Goto-Kakizaki rats. Am. J. Surg., 194 (2): 221-4, 2007.

5- BUCHWALD H., ESTOK R., FAHRBACH K. and BANEL D.: Weight and type 2 diabetes after bariatric surgery: Systematic review and meta-analysis. Am. J. Med., 122 (3): 248-56.e5, 2009.

6- HORWITZ D., SAUNDERS J.K., UDE-WELCOME A., MARIE SCHMIDT A., DUNN V., LEON PACHTER H., et al.: Three-year follow-up comparing metabolic surgery versus medical weight management in patients with type 2 diabetes and BMI 30-35. The role of sRAGE biomarker as predictor of satisfactory outcomes. Surg. Obes. Relat. Dis., 12 (7): 1337-41, 2016.

7- BUCHWALD H., ESTOK R., FAHRBACH K., BANEL D. and SLEDGE I.: Trends in mortality in bariatric surgery: A systematic review and meta-analysis. Surgery, 142 (4): 621-35, 2007

8- WEIR G. and BONNER-WEIR S.: Five stages of evolving beta-cell dysfunction during progression to diabetes. Diabetes, 53 (3): S16-21, 2004.

9- SHAH S.S., TODKAR J.S., SHAH P.S., CUMMINGS D.E. and SLEDGE I.: Diabetes remission and reduced cardiovascular risk after gastric bypass in Asian Indians with body mass index. Surg. Obes. Relat. Dis., 6 (4): 3328, 2007.

10- LEE W., HUR K., LAKADAWALA M. and KASAMA K.: Gastrointestinal metabolic surgery for the treatment of diabetic patients: A multi-institutional international study. J. Gastrointest. Surg., 16 (1): 45-52, 2012.

11- CHIELLINI C., RUBINO F., CASTAGNETO M. and NANNI G.: The effect of bilio-pancreatic diversion on type 2 diabetes in patients with BMI $<35 \mathrm{~kg} / \mathrm{m}^{2}$. Diabetologia, 52 (6): 1027-30, 2009.

12-SKROUBIS G., KOURI N., MEAD N. and KALFARENTZOS F.: Long-term results of a prospective comparison of Roux-en-Y gastric bypass versus a variant of biliopancreatic diversion in a non-superobese population (BMI 35-50kg/m²). Obes. Surg., 24 (2): 197-204, 2014. 


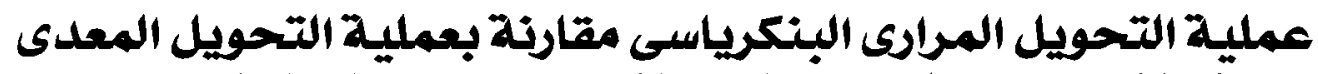

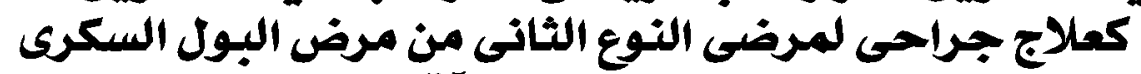

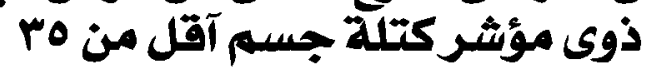

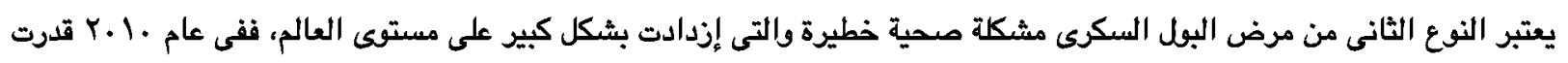

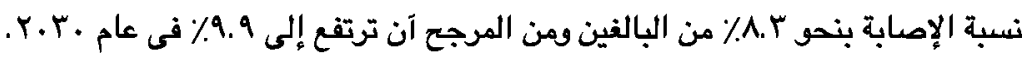

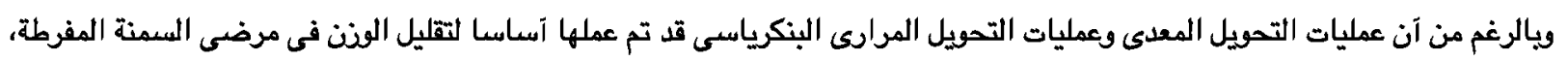

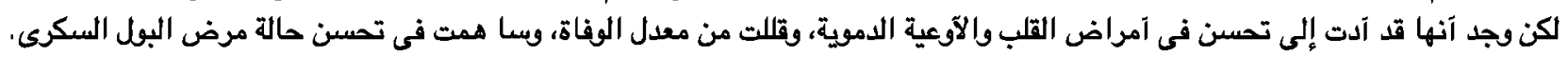

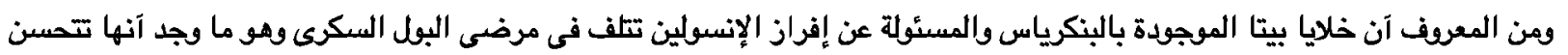

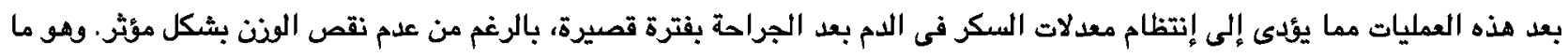
يبين وجود آليات آخرى لتحسن حالة السكر فى هؤلاء المرضى غير نير نقص الونن. هذه الآبحاث فى الإنسان تبشر بآمل جديد لمرضى هذا الداء المزمن. 